\title{
Polygalasaponin XXXII from Polygala tenuifolia root improves hippocampal-dependent learning and memory
}

\author{
Wei XUE, Jin-feng HU, Yu-he YUAN, Jian-dong SUN, Bo-yu LI, Dong-ming ZHANG, Chuang-jun LI, Nai-hong CHEN* \\ Institute of Materia Medica, Chinese Academy of Medical Sciences and Peking Union Medical College, Beijing 100050, China
}

\begin{abstract}
Aim: The aim of this study was to investigate the cognition-enhancing activity and underlying mechanisms of a triterpenoid saponin (polygalasaponin XXXII, PGS32) isolated from the roots of Polygala tenuifolia Willd.

Methods: The Morris water maze was used to evaluate the spatial learning and memory of mice. To detect the basic properties of synaptic transmission and long-term potentiation (LTP) in the dentate gyrus of rats, electrophysiological recordings were made of evoked potentials. Western blotting analysis and immunofluorescence assays were used to determine the phosphorylation of extracellular signal-regulated kinase (ERK), cAMP response element-binding protein (CREB), synapsin I and the expression of brain derived neurotrophic factor (BDNF).

Results: When administered at 0.125, 0.5, or $2 \mathrm{mg} / \mathrm{kg}$, PGS32 could significantly prevent scopolamine-induced cognitive impairments in mice. Intracerebroventricular (icv) administration of PGS32 greatly enhanced basic synaptic transmission in the dentate gyrus of rats and induced LTP. In primary hippocampal neurons, as well as in the hippocampus of maze-trained mice, PGS32 activated the mitogen-activated protein (MAP) kinase cascade by promoting phosphorylation of ERK, CREB and synapsin I. The expression of BDNF was also greatly enhanced in the hippocampus.

Conclusion: Our findings suggest that PGS32 can improve hippocampus-dependent learning and memory, possibly through improvement of synaptic transmission, activation of the MAP kinase cascade and enhancement of the level of BDNF. Therefore, PGS32 shows promise as a potential cognition-enhancing therapeutic drug.
\end{abstract}

Keywords: Polygala tenuifolia Willd; triterpenoid saponin; learning and memory; synaptic transmission; MAP kinase signaling system; brain derived neurotrophic factor

Acta Pharmacologica Sinica (2009) 30: 1211-1219; doi: 10.1038/aps.2009.112; published online 17 August 2009

\section{Introduction}

Cognitive dysfunction and dementia are symptoms of many diseases of the central nervous system and they represent major problems that can endanger the health and quality of life of people in contemporary society. Therefore, extensive searches are being carried out for novel, naturally occurring active compounds that can be used to treat multiple-factor dementia. Numerous medicinal plant species are now being screened for advanced therapeutic drugs that possess both high efficacy and low side effects ${ }^{[1-5]}$. One such plant, Polygala tenuifolia Willd, is the species of interest in this study.

The root of Polygala tenuifolia Willd, or "yuanzhi", is a wellknown traditional Chinese medicine that has been prescribed for thousands of years for a range of nervous system distur-

\footnotetext{
* To whom correspondence should be addressed.

E-mail chennh@imm.ac.cn

Received 2009-03-19 Accepted 2009-06-22
}

bances; these include amnesia, neurasthenia, palpitation, and insomnia. More recently, there has been renewed interest in the cognition-enhancing potential of $P$ tenuifolia root extracts. Several studies have shown that $P$ tenuifolia extracts have cognition improving effects in vivo, in addition to protective effects on neurotoxicity that is induced in vitro by factors such as glutamate, beta-amyloid, and the C-terminal fragment of amyloid precursor protein $(\mathrm{CT})^{[2,4-6]}$. However, the biologically active components from these extracts still require identification, and their in vivo functions and mechanisms must be understood before $P$ tenuifolia root extracts can be developed into therapeutic drugs.

One biologically active substance that can be extracted from $P$ tenuifolia roots is the triterpenoid saponin, polygalasaponin XXXII (PGS32). This substance was first isolated by Zhang et al in $1996^{[7]}$. Because the general saponins from $P$ tenuifolia root have shown cognition-enhancing effects, the aim of the present study is to investigate whether the specific saponin 
PGS32 shows any cognition-enhancing activity. Particular attention will be given to the possible effects on the hippocampus, because this is the region of the brain that is most related to learning and memory.

In mice, spatial learning is hippocampus-mediated ${ }^{[8]}$; consequently, behavioral tests that assess spatial learning, such as the Morris water maze test, can be used to investigate hippocampus-dependent learning and memory.

Long-term increases in synaptic efficacy, or long-term potentiation (LTP), can be observed in several brain regions including the hippocampus ${ }^{[9,10]}$, and this phenomenon is thought to be the major neurophysiological basis for the development of learning and memory. LTP is involved in learning behavior, and LTP in the dentate gyrus (DG) of the hippocampal formation is implicated in associative learning ${ }^{[11]}$. For these reasons, electrophysiological LTP assays were employed in the current study as a tool for screening our candidate cognition-enhancing drug.

Along with learning and memory, synaptic plasticity is now believed to be linked to the extracellular signal-regulated kinase/mitogen-activated protein kinase (ERK/MAPK) signaling pathway; this is a highly conserved kinase cascade that links transmembrane receptors to downstream effector mechanisms ${ }^{[12,13]}$. In rodents, behavior and memory, including spatial learning ${ }^{[14,15]}$ and conditioned place preference ${ }^{[16]}$, appear to involve the ERK/MAPK pathway.

Another key protein that is induced upon activation of learning and memory processes is brain derived neurotrophic factor (BDNF). This member of the neurotrophin family is expressed in brain areas that exhibit a high degree of plasticity (ie the hippocampus, and cerebral cortex). As such, BDNF is considered a genuine molecular mediator of functional and morphological synaptic plasticity ${ }^{[17]}$, and its induction can be a useful molecular marker for the activation of learning processes.

Based on these ideas, the aim of the current study was to investigate whether PGS32 had cognition-enhancing effects on the mouse scopolamine-induced amnesia model. Basic investigations on the action of PGS32 on synaptic transmission were extended to the molecular level by examining alterations in the MAP kinase cascade and in BDNF expression in the hippocampus.

\section{Materials and methods Drugs and reagents}

Scopolamine hydrobromide was purchased from the National Institute for the Control of Pharmaceutical and Biological Products (Beijing, China). Huperzine-A (HupA) was obtained from Sigma Chemicals (St Louis, MO, USA).

Anti-phospho-ERK1/2, anti- $\beta$-actin, anti-BDNF primary antibodies, and anti-rabbit IgG secondary antibody were purchased from Santa Cruz Biotechnology (Santa Cruz, CA, USA). Anti-phospho-CREB and anti-phospho-synapsinI antibody were obtained from Cell Signaling Biotechnology (Hertfordshire, England). Enhanced chemiluminescent (ECL) substrate was from Pierce (Rockford, IL, USA).

\section{Compound preparation}

The compound was prepared as previously described ${ }^{[18]}$. Briefly, dried roots of $P$ tenuifolia $(3.0 \mathrm{~kg})$ were powdered and extracted three times with $95 \%$ ethanol $(30 \mathrm{~L} \times 3)$ for $2 \mathrm{~h}$ each time. The solvent was then removed using reduced pressure; this yielded $0.68 \mathrm{~kg}$ residue. This residue was applied to a silica-gel column chromatography $(100-200$ mesh, $15 \times 50 \mathrm{~cm})$ and then successively eluted with $\mathrm{CHCl}_{3}(5 \mathrm{~L})$, ethyl acetate (5 L), acetone (5 L), and methanol (6 L). The methanol eluate $(380 \mathrm{~g})$ was applied to a macroporous resin (D101, $2 \mathrm{~kg}$, $15 \times 105 \mathrm{~cm})$ column and eluted with $\mathrm{H}_{2} \mathrm{O}(15 \mathrm{~L}), 30 \%$ ethanol (15 L), 60\% ethanol (15 L), and 95\% ethanol (15 L) (Fraction-a -Fraction-d). The $60 \%$ ethanol fraction (Fraction-c, $77.2 \mathrm{~g}$ ) was chromatographed on a silica gel column and eluted with $\mathrm{CHCl}_{3}$-methanol- $\mathrm{H}_{2} \mathrm{O}(70: 30: 5,6 \mathrm{~L}$ ) to yield ten fractions (Fraction c-1-Fraction c-10).

Fraction Fc-2 (6.0 g) was separated by preparative medium pressure liquid chromatography (MPLC), eluted with methanol- $\mathrm{H}_{2} \mathrm{O}$ (50:50, 2 L; 55:45, 4 L; 60:40, 4 L; 65:35, 4 L; 70:30, 4 L; $80: 20,2$ L) and purified by repeated semi-preparative high pressure liquid chromatography (HPLC) to yield compound polygalasaponin XXXII (PGS32).

\section{Animals}

Seventy-two male C57BL/6J mice (18-20 g), 8 weeks old, and twelve male Wistar rats (230-260 g), 3 months old, were obtained from the Experimental Animal Center of the Chinese Academy of Medical Sciences, Beijing. The mice were housed in groups of 4 in plastic wire-mesh cages $(30 \mathrm{~cm} \times 15 \mathrm{~cm} \times 12$ $\mathrm{cm})$, and the rats were housed in groups of 2 in plastic wiremesh cages $(50 \mathrm{~cm} \times 30 \mathrm{~cm} \times 18 \mathrm{~cm})$ in the animal house. Wood shavings used as beddings were replaced every 4 days. The animals had access to standard rodent chow and fresh tap water ad libitum. They were kept under a $12 \mathrm{~h}$ light: $12 \mathrm{~h}$ dark cycle (lights on: 08:00-20:00 h and lights off: 20:00-08:00 h). All experimental procedures on mice and rats were conducted during the light cycle. The average room temperature and relative humidity were kept at $22 \pm 2{ }^{\circ} \mathrm{C}$ and $55 \% \pm 5 \%$, respectively. The study was conducted in accordance with the internationally accepted principles for laboratory animal use and care (NIH publication № 86-23 revised 1985).

\section{Behavioral test \\ Drug administration}

According to their weight and autonomic activities, seventytwo C57BL/6J mice were divided into six groups (12 animals per group): a control group, model group, PGS32 groups, and positive control group. PGS32 was suspended in double distilled water (DDW) and administered orally to mice on a daily basis (PGS32 groups) at doses of $0.125,0.5$, or $2 \mathrm{mg} / \mathrm{kg}$. HupA $(0.05 \mathrm{mg} / \mathrm{kg})$ was suspended in DDW and orally administered (ig) to the positive control group. The same volumes $(0.1 \mathrm{~mL} / 10 \mathrm{~g})$ of DDW were given to the control group and the model group. The administration was conducted once at 8:00 from a period of $10 \mathrm{~d}$ prior to initiation of the behavioral experiments to the end of the water maze task. Thus, the total 
period of administration was 16 days. During the behavioral testing, the three PGS32 groups, the model group and the HupA group were injected with scopolamine $(1 \mathrm{mg} / \mathrm{kg}$ ip, suspended in saline) $30 \mathrm{~min}$ prior to the test, whereas the control group was injected with the same volume of normal saline (NS). The groups and treatment conditions are detailed in Table 1.

\section{Morris water maze}

The spatial learning and memory of mice was evaluated using the Morris water maze. The maze (Institute of Materia Medica, Chinese Academy of Medical Sciences, Beijing, China) was a dark circular pool, $120 \mathrm{~cm}$ in diameter and $55 \mathrm{~cm}$ high, filled to a depth of $25 \mathrm{~cm}$ with water $\left(22 \pm 1^{\circ} \mathrm{C}\right)$, which was made to be opaquely white with non-fat milk powder. A clear Plexiglas goal platform (10 cm in diameter) was located $1.5 \mathrm{~cm}$ below the surface in a constant position in the center of one quadrant. Every spatial cue external to the maze was held constant throughout the testing period. One $40 \mathrm{~W}$ daylight lamp was positioned on the table beside the arena and aimed at the ceiling to indirectly illuminate the surface of the water. To monitor the subject's swimming parameters, a closed-circuit television camera linked to a computer-based image analyzer was mounted onto the ceiling directly above the center of the pool.

The training trials began on the 11th day of the intragastric administration; they consisted of two consecutively repeated trials per day for a period of $5 \mathrm{~d}$. All testing began at 10:00. Each mouse was placed in the water, facing the wall of the tank at the starting point, and allowed to swim until they found the hidden platform that was located in the maze. During each trial, a mouse was given $90 \mathrm{~s}$ to find the hidden platform. If it remained in the water after $90 \mathrm{~s}$, the mouse was gently guided to and placed on the platform. It was then allowed to stay on the platform for $15 \mathrm{~s}$ before a similar second trial was initiated. After the trials, the mouse was removed to its cage and placed under a $60 \mathrm{~W}$ lamp to dry and warm for 5 min. The time required for the mouse to reach the platform (escape latency) was recorded. On the sixth day, the mice were subjected to a probe trial session in which the platform was removed from the pool, and they were allowed to swim for $90 \mathrm{~s}$ to search for it. A record was kept of the swimming time in the pool quadrant where the platform had been previ- ously placed.

Six mice in each group were decapitated immediately following the behavioral test. The brains were removed and the hippocampal formations were dissected out, snap-frozen in liquid nitrogen, and stored at $-80{ }^{\circ} \mathrm{C}$ until required for further analysis of the levels of phospho-ERK, phospho-CREB, phospho-synapsin I and BDNF.

\section{Electrophysiological assays Electrophysiological recording}

Recordings of evoked potentials were made as previously described $^{[19,20]}$. Briefly, male Wistar rats were anesthetized with urethane carbamate $(1.5 \mathrm{~g} / \mathrm{kg}$, ip) before being fixed on a SR-6N stereotaxic apparatus (Narishige, Japan). Three holes were sequentially drilled at $0.8 \mathrm{~mm}, 3.8 \mathrm{~mm}$, and $7.5 \mathrm{~mm}$ posterior to Bregma and $1.8 \mathrm{~mm}, 2.5 \mathrm{~mm}$, and $4.2 \mathrm{~mm}$ lateral to the mid-line to allow placement of an outer guide cannula, a monopolar recording electrode and a bipolar stimulating electrode, respectively. The cannula was placed into the lateral cerebral ventricle at a depth of $2.5-3.0 \mathrm{~mm}$, the recording electrode was placed in the granular cell layer of dentate gyrus at a depth of 3.0-3.5 $\mathrm{mm}$, and the stimulating electrode was lowered into the perforant path (PP) to a depth of 3.0-3.5 mm. The synaptic responses were monitored on a VC-11 memory oscilloscope (Nihon Kohden, Japan).

The amplitude of population spike (PS) was employed to assess the excitation level of the granular cell population in the dentate gyrus. An evoked response was generated in the granular cell layer by stimulating the PP at low frequency $(0.033 \mathrm{~Hz})$ with single constant current pulses (150 $\mu$ s in duration) triggered by a SEN-7203 electrical stimulator (Nihon Kohden, Japan) through a SS-202J isolator (Nihon Kohden, Japan). After input/output curve determination, the baseline responses were evoked by a stimulus with an intensity that produced $20 \%$ of the maximal PS amplitude.

\section{Intracerebroventricular (i.c.v.) drug delivery}

Twelve Wistar rats (230-260 g), 3 months old, were randomly assigned to either a control group $(n=6)$ or a PGS32 group $(n=6)$. Electrophysiological baseline measurements from the dentate gyrus of the contralateral hemisphere were measured for 30 min. Subsequently, control rats were injected with $0.4 \%$ dimethyl sulfoxide (DMSO), whereas PGS32 rats were injected

Table 1. Groups and treatment conditions in the Morris water maze test.

\begin{tabular}{|c|c|c|}
\hline Treatment groups & po $(\mathrm{d} 1-\mathrm{d} 16)$ & ip (d 11-d 16) \\
\hline Control group & Vehicle (DDW $)^{\mathrm{a}}$ & Vehicle (saline) ${ }^{\mathrm{b}}$ \\
\hline Model group & Vehicle (DDW) $)^{\mathrm{a}}$ & $1 \mathrm{mg} / \mathrm{kg}$ scopolamine ${ }^{\mathrm{b}}$ \\
\hline 0.125 mg/kg PGS32 group & 0.125 mg/kg PGS32 & $1 \mathrm{mg} / \mathrm{kg}$ scopolamine $\mathrm{b}^{\mathrm{b}}$ \\
\hline 0.5 mg/kg PGS32 group & $0.5 \mathrm{mg} / \mathrm{kg}$ PGS32 & $1 \mathrm{mg} / \mathrm{kg}$ scopolamine $\mathrm{b}^{\mathrm{b}}$ \\
\hline 2 mg/kg PGS32 group & $2 \mathrm{mg} / \mathrm{kg}$ PGS32 & $1 \mathrm{mg} / \mathrm{kg}$ scopolamine \\
\hline Positive control group & 0.05 mg/kg Huperzine- $\mathrm{A}^{\mathrm{a}}$ & $1 \mathrm{mg} / \mathrm{kg}$ scopolamine $\mathrm{b}^{\mathrm{b}}$ \\
\hline
\end{tabular}

${ }^{a}$ Administered once daily ig from $10 \mathrm{~d}$ before testing till end of behavioral testing; ${ }^{b}$ Administered ip 30 min before testing 
with $400 \mu \mathrm{mol} / \mathrm{L}$ PGS32 (in $0.4 \%$ DMSO) via a cannula in the lateral cerebral ventricle. Injections were performed using a 5 $\mu \mathrm{L}$ volume over a $5 \mathrm{~min}$ period via a Hamilton syringe. The dose of PGS32 was calculated based on the theoretical concentration that the drug would have in the brain (assuming a brain volume of approximately $2 \mathrm{~mL}$ ). Thus, for an estimated brain concentration of PGS32 at $1 \mu \mathrm{mol} / \mathrm{L}, 5 \mu \mathrm{L}$ of $400 \mu \mathrm{mol} / \mathrm{L}$ was injected.

\section{Data collection and analysis}

Each time point measurement represented the mean of five evoked responses. The mean baseline was obtained by averaging the PS amplitudes of six time points taken within the 30 min period prior to the icv injection of drugs or vehicle. The data for each time point were expressed as a mean percentage of the mean baseline. The differences in the baseline between and within the groups and the differences between the groups after administration were analyzed using two-way analysis of variance (ANOVA) with repeated measures.

\section{Western blotting analysis}

After Morris water maze testing, six mice from each group were decapitated and hippocampus samples from the mice were homogenized thoroughly and then lysed in a RIPA lysis buffer (50 mmol/L Tris (pH 7.4), $150 \mathrm{mmol} / \mathrm{L} \mathrm{NaCl}, 1 \% \mathrm{NP} 40$, $0.5 \%$ sodium deoxycholate and $0.1 \%$ SDS). Protein concentrations were measured with a BCA kit (Pierce). The sample lysates were solubilized in SDS sample buffer, separated by SDS-PAGE gel, and transferred to a PVDF membrane (Millipore). The membrane was blocked with 3\% BSA (Sigma) and incubated with primary antibody, followed by horseradish peroxidase (HRP)-conjugated secondary antibody. Detection was performed using an enhanced ECL plus detection system (Molecular Devices, Lmax). The relative density of the bands was analyzed by Gelpro 32 software. Levels of phospho-ERK, phospho-CREB, phospho-synapsin I, and BDNF were calculated in the samples from control, model, PGS32 and HupA groups.

\section{Cell cultures and immunofluorescence analysis}

Primary cultures of hippocampal cells were established using a modification of the procedure of Banker and Cowan (1977). The day before plating, plastic culture dishes containing glass coverslips were treated with poly-L-lysine $(100 \mathrm{mg} / \mathrm{mL})$. On the day of plating, newborn Wistar rats (P0) were killed using $\mathrm{CO}_{2}$ and cervical dislocation. The hippocampi were then dissected out and incubated for $10 \mathrm{~min}$ in an enzymatic solution ( $0.125 \%$ trypsin and $1 \mathrm{mmol} / \mathrm{L}$ EDTA in Hanks's solution), washed three times in minimal essential medium (MEM), and triturated to dissociate cells. Four hundred microliters of cell suspension (diluted to $1 \times 10^{6}$ cells $/ \mathrm{mL}$ ) was added to poly- $L$ lysine coated dishes and coverslips. Cultures were placed in an incubator $\left(5 \% \mathrm{CO}_{2}, 37^{\circ} \mathrm{C}\right)$. To prevent glial proliferation, $10 \mu \mathrm{mol} / \mathrm{L}$ Ara-C was added to the cultures at 3 DIV.

At 5 DIV, the hippocampal neurons were prepared for immunofluorescence assay using a Leica TCSNT confocal fluorescence microscope with a $\times 20$ lens (Wetzler, Heidelberg, Germany). The dishes were randomly divided into control groups and PGS32 groups. PGS32 $(1 \mu \mathrm{mol} / \mathrm{L}$ in $0.001 \%$ DMSO-PBS) was added to the PGS32 group and incubated in water at $37^{\circ} \mathrm{C}$ for exactly $4 \mathrm{~min}$. Vehicle (0.001\% DMSO-PBS) was added to the control group under the same conditions. The cells were rapidly washed with PBS and fixed by paraformaldehyde. After fixation, the cells were permeabilized with $0.2 \%(v / v)$ Triton X-100 and then blocked with 3\% goat serum. The cells were then incubated at $4{ }^{\circ} \mathrm{C}$ overnight with a 1:100 dilution of phospho-ERK1/2, phospho-CREB, or phospho-synapsin I antibody. The neurons were incubated for 30 min with a 1:100 dilution of fluorescein isothiocyanate-conjugated goat anti-rabbit IgG and rhodamine-labeled phalloidin. Immunoreactivity was monitored using a Leica TCSNT confocal fluorescence microscope.

\section{Statistical analysis}

Data were expressed as means \pm SEM as indicated. Group differences of escape latency in training trials of the Morris water maze test and PS amplitude in the electrophysiological assays were analyzed using two-way analysis of variance (ANOVA) with repeated measures. Group differences in the probe trial and biochemical assays were evaluated using one-way ANOVA followed by Duncan's multiple-range test. $P$-values of $<0.05$ and 0.01 were regarded as statistically significant.

\section{Results}

\section{Effects of PGS32 on performance in the Morris water maze test}

The effects of PGS32 $(0.125,0.5$, and $2 \mathrm{mg} / \mathrm{kg})$ on the scopolamine-induced impairment of spatial learning and memory were evaluated using the Morris water maze test. In naive mice, the mean latency in finding the hidden platform declined progressively during the training period of 5 consecutive days (Figure 1A). The mice in the model group treated with scopolamine $(1 \mathrm{mg} / \mathrm{kg}$, ip) showed impaired spatial learning and memory, as indicated by a longer latency to find the platform than the control group. Two-way ANOVA (group $\times$ days, $6 \times 5$ ) with repeated measures revealed a significant difference in the main effect between the control and model groups $[F(1,22)=49.310, P<0.001]$ and significant interaction $[F(4,19)=4.327, P<0.001]$. In contrast, the prolonged latency was significantly shortened in the mice of the 0.125 $\mathrm{mg} / \mathrm{kg}[F(1,22)=12.657, P<0.01]$ and $2 \mathrm{mg} / \mathrm{kg}$ PGS32 groups $[F(1,22)=4.757, P<0.05]$ compared with the model group. There was also a trend toward shorter latency in the PGS32 $0.5 \mathrm{mg} / \mathrm{kg}$ group $[F(1,22)=4.179, P=0.053]$. Likewise, HupA $(0.05 \mathrm{mg} / \mathrm{kg})$ pretreatment yielded similar improvements $[F(1,22)=6.279, P<0.05]$.

In the probe trial session, swimming times within the target quadrant of the model group were significantly shorter than those of the control group (Figure 1B, $P<0.01$ ). Moreover, the reduced swimming times induced by scopolamine were significantly increased by $0.125,0.5,2 \mathrm{mg} / \mathrm{kg}$ PGS32 and HupA $(P<0.05,0.01,0.05,0.05$, respectively), and the swimming speed was not significantly different between each group (data 

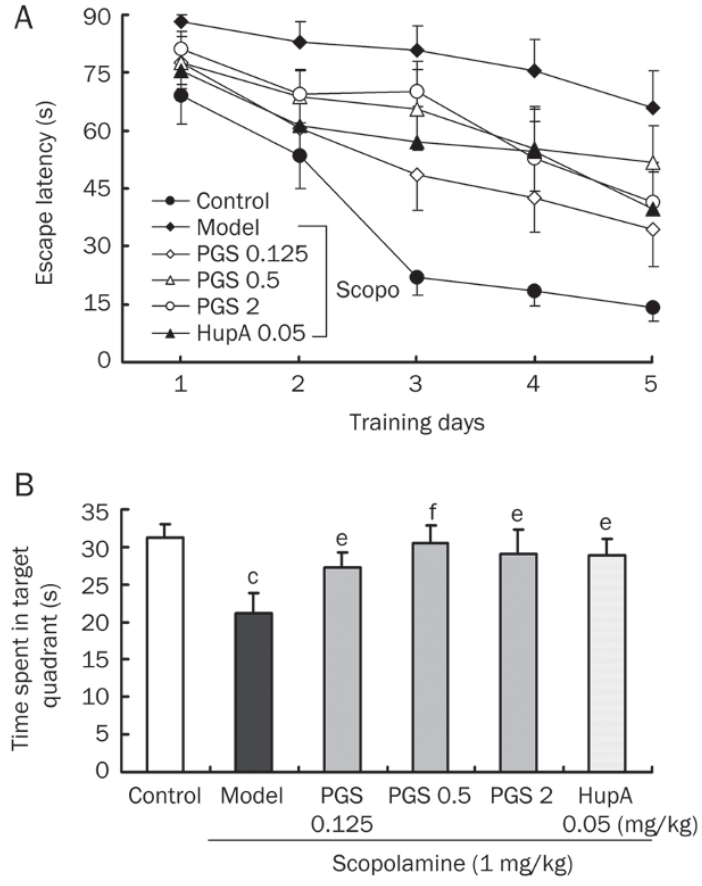

Figure 1. Effects of PGS32 on performance in the training trials (A) and in the probe trial (B) of the Morris water maze task in mice with scopolamine-induced cognitive deficits. Data represent means \pm SEM $(n=12)$. Mice were orally treated with PGS32 $(0.125,0.5$, and $2 \mathrm{mg} / \mathrm{kg})$, the same volume of saline, or huperzine-A (Hup A, $0.05 \mathrm{mg} / \mathrm{kg}$ ) for 16 days for a period ranging from $10 \mathrm{~d}$ prior to beginning the behavioral experiments to the end of the water maze task. Scopolamine $(1 \mathrm{mg} / \mathrm{kg})$ or the same volume of saline was administered ip to mice $30 \mathrm{~min}$ before the training trial and the probe trial. (A) Mean escape latency to find the hidden platform over 5 consecutive days of the training trial. The data were analyzed using two-way ANOVA (group $\times$ days, $6 \times 5$ ) with repeated measures. (B) Swimming time in the target quadrant in $90 \mathrm{~s}$ probe trial (no platform). Each mouse was subjected to $90 \mathrm{~s}$ observation on the 6 th day of the water maze tasks. ${ }^{c} P<0.01$ vs control group. ${ }^{e} P<0.05,{ }^{\text {f }} P<0.01$ vs model group.

not shown).

Effects of PGS32 on basic synaptic transmission in the dentate gyrus of anesthetized rats

The population spike was evoked to provide a measure of the excitability of neurons (Figure 2A, 2B, and 2C). When provided with low-frequency stimulation, the baseline amplitude of the PS stabilized within the first $30 \mathrm{~min}$ in both the control and the PGS32 groups; two-way ANOVA showed no significant difference between the groups $[F(1,10)=1.860, P=0.203]$, as well as the six time points $[F(5,6)=1.242, P=0.394]$ (Figure 2D). Moreover, the PS amplitude did not fluctuate significantly for $60 \mathrm{~min}$ after vehicle (0.4\% DMSO-PBS) administration $[F(5,6)=7.909, P=0.263]$. In contrast, after $i c v$ administration of $1 \mu \mathrm{mol} / \mathrm{L}$ PGS32, the PS amplitude increased to $162 \% \pm 13 \%$ of baseline within $5 \mathrm{~min}$. By 10, 30, and $60 \mathrm{~min}$, the PS amplitude had increased to $172 \% \pm 8 \%, 215 \% \pm 17 \%$, and $254 \% \pm 32 \%$ of baseline, respectively. There was a significant main effect of
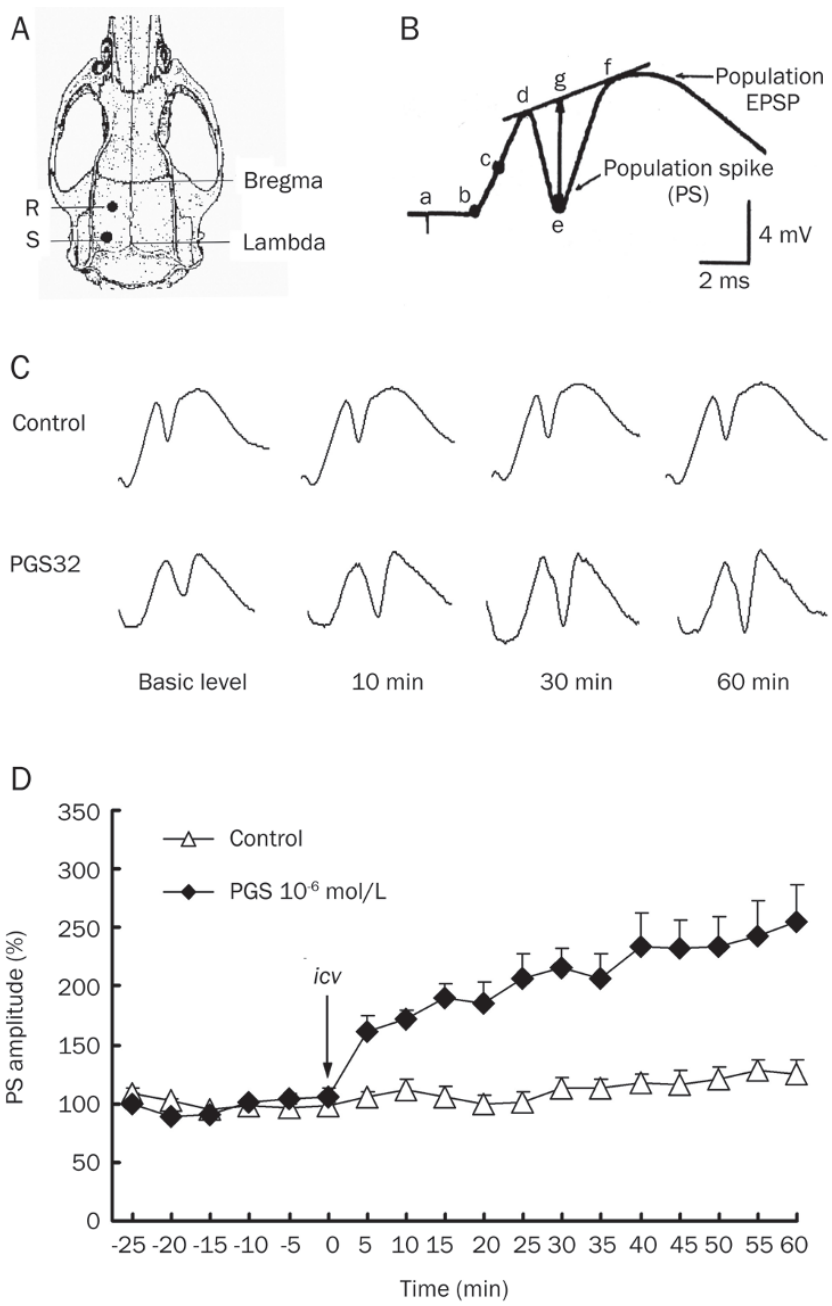

Figure 2. Effects of PGS32 on basic synaptic transmission in the DG of anesthetized rats. (A) Anatomical diagram of a rat skull. The points $\mathrm{R}$ and $S$ represent the approximate location of the recording electrode and the stimulating electrode, respectively. (B) Measurement of PS amplitude. a: stimulus artifact; b: initial point of excitatory postsynaptic potential (EPSP); c: middle point of bd; $d, e, f$ : end points of the peaks; length of ge: amplitude of PS. (C) Original figure of PS in control and PGS32 group at baseline, $10 \mathrm{~min}, 30 \mathrm{~min}$, and $60 \mathrm{~min}$ after icv. (D) Time-course plots of perforant path-evoked population spikes (PS) of the control group (white triangle) and PGS32 group (black rhombus). The stimulating electrode and the recording electrode were placed within the perforant path (PP) and the granular cell layer of the dentate gyrus, respectively. An evoked response was generated in the dentate gyrus granular cell layer by stimulating the PP at low frequency $(0.033 \mathrm{~Hz})$; PS of the granular cell layer in DG was recorded throughout the test. The baseline was recorded from $30 \mathrm{~min}$ before administration. At $0 \mathrm{~min}, 5 \mu \mathrm{L} 400 \mu \mathrm{mol} / \mathrm{L}$ PGS32 (icv, final concentration was $1 \mu \mathrm{mol} / \mathrm{L}$ ) was injected in the test subject, whereas $0.4 \%$ DMSO-PBS was injected in a control animal.

the groups $[F(1,10)=15.876, P<0.01]$. Furthermore, the increase in the PS amplitude of more than $30 \%$ over baseline (which lasted for more than $30 \mathrm{~min}$ ) indicated a PGS32-induced LTP in the hippocampal dentate gyrus. 
Effects of PGS32 on the phosphorylation of ERK, CREB, synapsin $\mathrm{I}$, and the levels of BDNF in the hippocampus of trained mice In order to elucidate the mechanism of the apparent cognitionenhancing effect observed by PGS32 in the maze test, we investigated changes at the molecular level, including activity of the MAP kinase cascade and expression of BDNF in the trained mice (Figure 3). No significant differences were observed in the phosphorylation levels of ERK in the hippocampal formations of mice from the control and model groups. In contrast, in PGS32 treated mice, significant increases in ERK phosphorylation were detected $(P<0.05,0.01$ vs model; $P<0.05,0.01$ vs control). In addition, phosphorylation of the transcription factor CREB was significantly decreased in the model group compared with the control group ( $P<0.05$ vs control), whereas the levels of hippocampal phospho-CREB were substantially increased in the PGS32-treated animals, especially at doses of $2 \mathrm{mg} / \mathrm{kg}(P<0.05,0.01$ vs model; $P<0.05,0.01$ vs control).

A

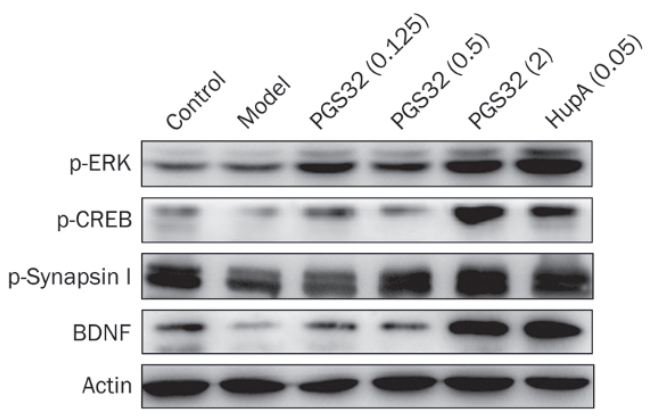

B

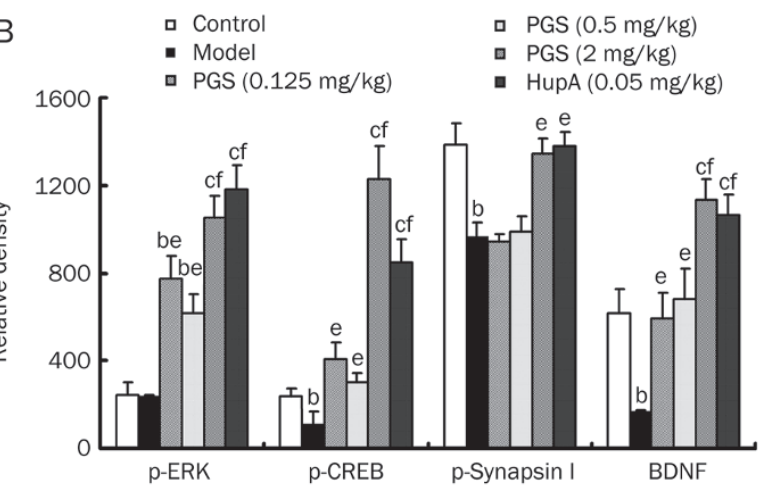

Figure 3. Phosphorylation of ERK, CREB, and synapsin I and the expression of BDNF of hippocampal lysates of mice following the Morris water maze task $(n=6)$. (A) Representative immunoblots of hippocampal lysates of the mice. The mice were administered and trained for the water maze tasks, and then hippocampus samples from six mice of each group were homogenated for immunoblotting. (B) The relative density of the bands was analyzed using one-way ANOVA. The six columns from the left to the right in each protein represent the control group, model group, PGS32 $0.125,0.5,2 \mathrm{mg} / \mathrm{kg}$ group and HupA group, respectively. ${ }^{\mathrm{b}} P<0.05,{ }^{\mathrm{c}} P<0.01$ vs control group. ${ }^{\mathrm{e}} P<0.05,{ }^{\mathrm{f}} P<0.01$ vs model group.

One of the roles of CREB is to regulate the expression of BDNF, a key protein involved in neuronal plasticity and the formation of memory. In the model group, hippocampal levels of BDNF were significantly reduced compared with those of the control animals $(P<0.05$ vs control). However, in PGS32 treated animals, the levels of BDNF were greatly increased, especially at a dose of $2 \mathrm{mg} / \mathrm{kg}(P<0.05,0.01 \mathrm{vs}$ model; $P<0.05$ vs control). These responses reflected the observed differences in CREB phosphorylation. The phosphorylation of synapsin I was also attenuated in the scopolamine group, and this attenuation was reversed by PGS32, especially at a dose of $2 \mathrm{mg} / \mathrm{kg}$ ( $P<0.05$ vs model).

In the HupA positive control group, phosphorylation of ERK, CREB, synapsin I, and expression of BDNF was increased $(P<0.05,0.01$ vs model), which suggested that HupA also improved cognition via a mechanism similar to that of PGS32.

Effects of PGS32 on the phosphorylation of ERK1/2, CREB, and synapsin I in primary hippocampal neurons

In addition to studying the effects of PGS32 on the MAP kinase cascade in C57 mice, we also investigated its effects on primary hippocampal neurons incubated with either 1 $\mu \mathrm{mol} / \mathrm{L}$ PGS32 or vehicle (0.001\% DMSO-PBS). After a $4 \mathrm{~min}$ treatment period, the neurons were fixed and phosphorylation of ERK, CREB, and synapsin I was measured. As shown in Figure 4, the green fluorescence intensity, which indicated the levels of phospho-ERK and phospho-CREB, were substantially increased in the plasma of neurons from the PGS32 group. Phospho-synapsin I increased in both the plasma membrane and the synapses of neurons from the PGS32 group. The results indicate that PGS32 can initiate rapid activation (in as little as $4 \mathrm{~min}$ ) of these critical proteins in primary hippocampal neurons.

\section{Discussion}

Recent reports of the cognition-improving activity of crude extracts of Polygala tenuifolia Willd have given support to the long understood cognition-enhancing activity of this plant, which was recorded in ancient Chinese medical books. However, the exact nature of the active ingredients in Polygala tenuifolia Willd has not yet been clarified. Therefore, in order to develop and utilize this traditional medicine as a clinical therapy for improving cognition deficits, the pharmacology and mode of action of its active components must be further investigated.

Spatial cognition is one hippocampus-mediated function that appears to be compromised in the elderly and in patients suffering from dementia. It is thought that these deficits can be at least partly modeled by the administration of scopolamine to normal adult animals ${ }^{[21]}$. In the current study, PGS32 at dosages of 0.125 and $2 \mathrm{mg} / \mathrm{kg}$ improved scopolamineinduced spatial cognitive deficits. This suggests that its cognition-enhancing effect observed in these animal models may translate to beneficial effects on human patients suffering from dementia in clinical settings. In the $0.5 \mathrm{mg} / \mathrm{kg}$ PGS32 group, there was no significant improvement of the latency in the training trials of the water maze test $(P=0.053)$. However, the swimming time in the target quadrant was greatly pro- 

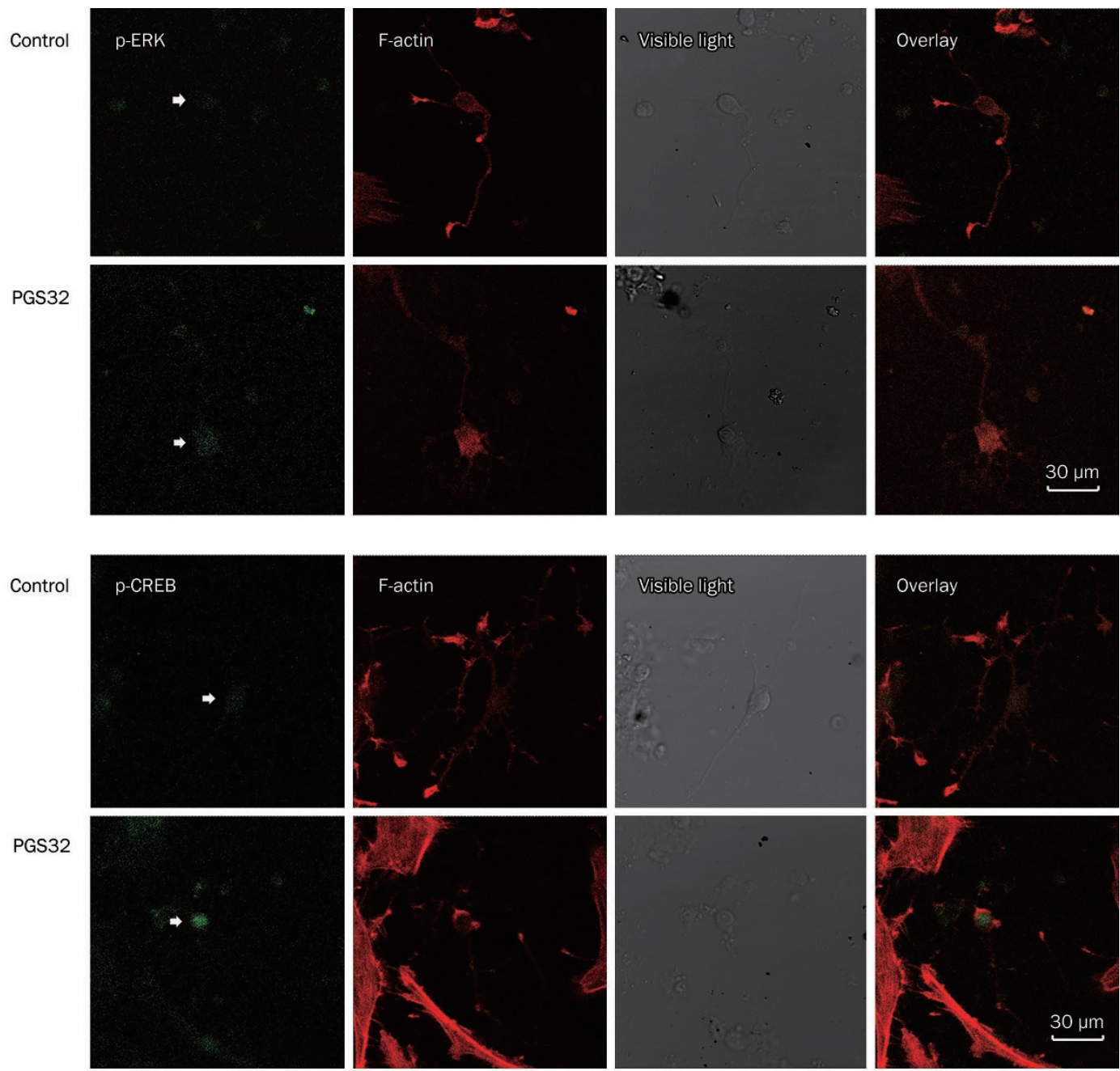

Figure 4. Effects of PGS32 on the phosphorylation of ERK1/2, CREB, and synapsin I in primary hippocampal neurons. The 5 DIV neurons were incubated with either 1 $\mu \mathrm{mol} / \mathrm{L}$ PGS32 (PGS32 group)
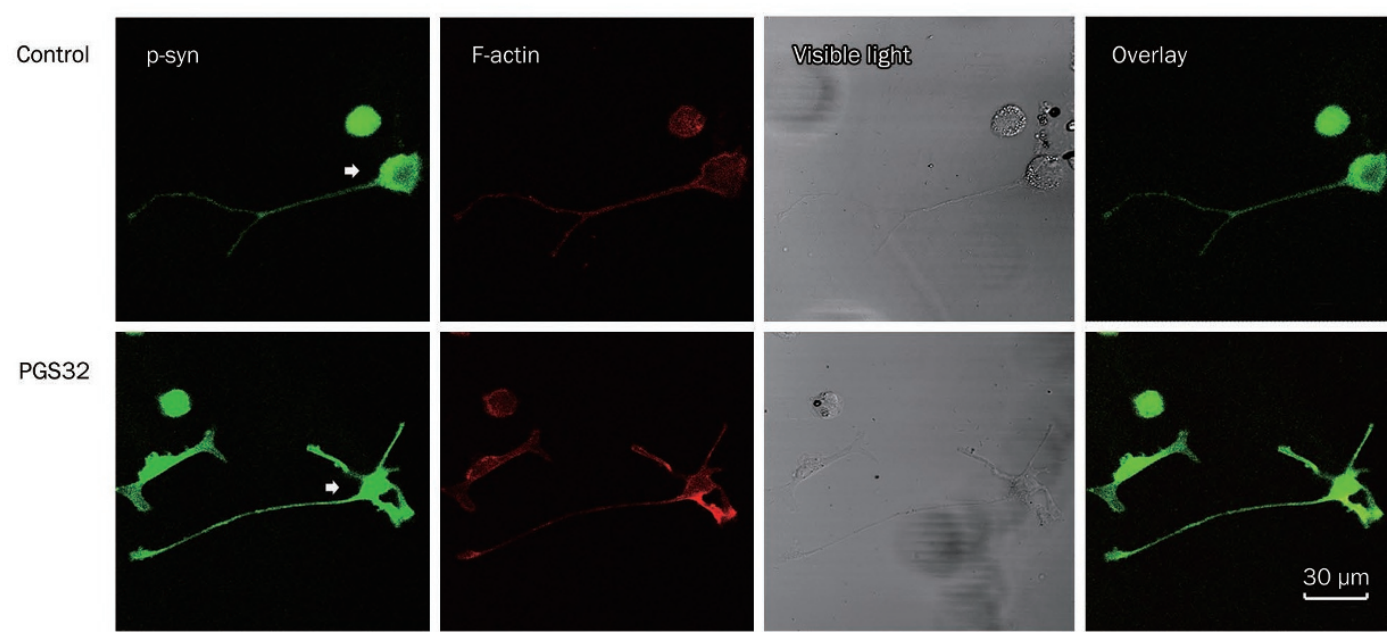
or vehicle $(0.001 \%$ DMSOPBS, Control group) at $37^{\circ} \mathrm{C}$ for $4 \mathrm{~min}$; cells were then fixed and phosphorylation of ERK, CREB, and synapsin I was detected $(n=3)$. The green fluorescence was used to label the levels of phosphoERK, phospho-CREB, and phospho-synapsin I in the control and PGS32 groups (arrow pointed). Rhodaminelabeled phalloidin was used to detect the F-actin of the neurons, so that their outline was displayed.

longed in the probe trial in this group; this may suggest that the sample size for behavioral testing should be increased and that further refinements to the dosages need to be considered. Huperzine A (HupA), a lycopodium alkaloid isolated from Huperzia serrata (Qian Ceng Ta), has been widely used for AD (Alzheimer's disease) therapy in China and has been shown to enhance memory, cognitive skills and daily life capabilities in both $\mathrm{AD}$ and VD (vascular dementia) patients ${ }^{[22]}$. Using HupA as a positive control, we discovered that the action of PGS32 on the cognitive function of scopolamine-treated mice was comparably good; this suggested that PGS32 has as much promise as a potential cognition-enhancing therapeutic drug 
as HupA.

LTP in the dentate gyrus (DG) of the hippocampal formation is implicated in spatial learning ${ }^{[23]}$, and this cellular model of hippocampal learning has been intensively studied at the perforant path-dentate granule cell synapse ${ }^{[24]}$. We confirmed the effect of PGS32 on hippocampal-dependent cognition using electrophysiological studies of basic synaptic transmission and LTP in the DG. We found that basic synaptic transmission was potentiated within $5 \mathrm{~min}$ and that LTP was induced by intracerebral ventricle injection of PGS32 at a concentration of 1 $\mu \mathrm{mol} / \mathrm{L}$. These results demonstrated a potentially novel effect of PGS32 on hippocampal-dependent learning and memory and suggested a mechanism that may underlie its action.

When the cognition-enhancing effects of PGS32 were investigated with respect to the effects on the ERK/MAPK signaling pathway and BDNF, further information regarding the mechanism of PGS32 at the molecular level was obtained. The ERK/MAP kinase cascade is important for spatial learning and synaptic plasticity, and its myriad of upstream signals converge once ERK has been activated ${ }^{[25]}$. In the current study, ERK was activated by PGS32 in primary hippocampal neurons as well as in the mouse hippocampus. Therefore, PGS32 presumably was able to prime the ERK/MAP kinase cascade by activating ERK. A large body of research has demonstrated that ERK activation is required for the induction and maintenance of LTP, as well as for hippocampal memory formation and retention ${ }^{[26,27]}$. The ability of PGS32 to activate ERK therefore provides initial evidence that this saponin can play a role in regulating cognitive function and synaptic transmission.

The CREB transcription factor is a substrate for a variety of protein kinases, including MAP kinase ${ }^{[28]}$. Evidence suggests that CREB activation is a critical step in the signaling cascade that initiates protein synthesis and the long-term morphological modifications that underlie LTP and long-term memory ${ }^{[29]}$. Consequently, agents that promote increases in the amounts or activity of CREB accelerate the process of long-term memory formation ${ }^{[30]}$. In the current study, PGS32 significantly increased the phosphorylation of CREB in primary hipppocampal neurons in as little as $4 \mathrm{~min}$, as well as in mouse hippocampal regions after administration for a period of 10 days and participation in spatial learning tasks. Therefore, the activation of CREB may be another key intermediate step underlying the mechanisms of cognition-deficit improvement and synaptic transmission potentiation that follows PGS32 treatment. However, the degree of involvement of ERK in the activation of CREB by PGS32 requires further study.

Increasing evidence indicates that BDNF plasma levels are significantly decreased in both elderly subjects and $\mathrm{AD}$ patients ${ }^{[17]}$; this suggests that a decrease in BDNF levels could be associated with $\mathrm{AD}$ pathogenesis ${ }^{[31]}$. In the current study, expression of BDNF was reduced in the model group treated with scopolamine, which provided a potent evidence that scopolamine treatment could imitate the state of cognition in aging people or AD patients. In addition, previous studies have shown that the cholinergic system is greatly involved in the regulation of BDNF mRNA ${ }^{[32,33]}$. Pilocarpine, a muscarinic agonist, markedly increased hippocampal BDNF mRNA levels in both early postnatal and adult rats, and this elevation could be completely antagonized by scopolamine. Accordingly, injected scopolamine down-regulated the expression of BDNF in our model group.

However, in the PGS32 groups the level of BDNF was not down-regulated by scopolamine treatment. It was much higher in the $2 \mathrm{mg} / \mathrm{kg}$ PGS32 group than in the control group, which suggested that PGS32 may regulate the expression of BDNF not only by stimulating the cholinergic system against scopolamine, but also by other mechanisms such as activation of the MAP kinase cascade. In this study, we have demonstrated that CREB in the MAP kinase cascade can be activated by PGS32. Because PGS32 is a key transfactor controlling the expression of $\mathrm{BDNF}^{[34]}$, it may also be the trigger that leads to the increased levels of BDNF in the PGS32 groups.

These molecular measurements were consistent with the performance of mice in behavioral studies and suggested that the improvement in hippocampal-dependent learning and memory occurred via MAP kinase cascade-activated BDNF expression.

On the other hand, BDNF is itself a signal promoter that can activate its receptor-TrkB and consequently stimulate the MAP kinase pathway. In other words, BDNF/TrkB signaling can converge on the MAP kinase pathway and can enhance both excitatory synaptic transmission in vivo and hippocampal-dependent learning in behaving animals ${ }^{[8]}$. Thus, a positive feedback loop can be established. Therefore, PGS32 may improve hippocampal-dependent learning and memory formation by activating ERK and CREB and further increasing the expression of BDNF. This in turn activates a BDNF/ TrkB/MAPK cascade and results in the consolidation of new memory functions.

Synapsin I is reported to be a downstream effector for the neurotrophin/TrkB/MAP kinase cascade. MAP kinasedependent phosphorylation of synapsin I may contribute to the modulation of neurotransmitter release and synaptic plasticity ${ }^{[35]}$. In the current study, phosphorylation of synapsin I was significantly increased in the plasma membrane and synapses of hippocampal neurons from the PGS32 group. This also occurred in mouse hippocampal regions following PGS32 treatment, which suggested that the neurotransmitter release might be up-regulated and synaptic plasticity might be improved. This may be taken as evidence of an effect of PGS32 on synaptic transmission in the DG.

In summary, the triterpenoid saponin PGS32, polygalasaponin XXXII, from the root of Polygala tenuifolia Willd improved hippocampal-dependent learning and memory in mice with scopolamine-induced amnesia. This occurred through improvements to basic synaptic transmission in the dentate gyrus of anesthetized rats, activation of the MAP kinase cascade and BDNF expression. The results from the current study clearly indicate that saponins from $P$ tenuifolia have tremendous potential for the development of therapeutic drugs that can be used to improve cognition in patients with cognitive deficit diseases. 


\section{Acknowledgments}

This work was supported in part by grants from Naitonal Natural Science Foundation of China (№ 30572342), the Program for Changjiang Scholars and Innovative Research Team in University (PCSIRT No IRT0514), and the basic R\&D Project for Central public research institutes-Youth Fund (2007CHX04).

\section{Author contribution}

Wei XUE designed the study, performed the research and wrote the paper; Jin-feng HU and Yu-he YUAN provided assistance in experiment methods; Jian-dong SUN and Bo-yu LI performed partial research; and Dong-ming ZHANG and Chuang-jun LI extracted the PGS32. Nai-hong CHEN designed the research and revised the paper.

\section{References}

1 Sakata Y, Chida R, Ishige K, Edagawa Y, Tadano T, Ito Y. Effect of a nutritive-tonic drink on scopolamine-induced memory impairment in mice. Biol Pharm Bull 2005; 28: 1886-91.

2 Sun XL, Ito H, Masuoka T, Kamei C, Hatano T. Effect of Polygala tenuifolia root extract on scopolamine-induced impairment of rat spatial cognition in an eight-arm radial maze task. Biol Pharm Bull 2007; 30: 1727-31.

3 Joshi $\mathrm{H}$, Parle M. Brahmi rasayana improves learning and memory in mice. Evid Based Complement Alternat Med 2006; 3: 79-85.

4 Park CH, Choi SH, Koo JW, Seo JH, Kim HS, Jeong SJ, et al. Novel cognitive improving and neuroprotective activities of Polygala tenuifolia Willdenow extract, BT-11. J Neurosci Res 2002; 70: 48492.

5 Ikeya Y, Takeda S, Tunakawa M, Karakida H, Toda K, Yamaguchi T, et al. Cognitive improving and cerebral protective effects of acylated oligosaccharides in Polygala tenuifolia. Biol Pharm Bull 2004; 27: 1081-5.

6 Naito R, Tohda C. Characterization of anti-neurodegenerative effects of Polygala tenuifolia in Abeta(25-35)-treated cortical neurons. Biol Pharm Bull 2006; 29: 1892-6.

7 Zhang D, Miyase T, Kuroyanagi M, Umehara K, Ueno A. Five new triterpene saponins, polygalasaponins XXVIII-XXXII from the root of Polygala japonica Houtt. Chem Pharm Bull (Tokyo) 1996; 44: 810-5.

8 Tyler WJ, Alonso M, Bramham CR, Pozzo-Miller LD. From acquisition to consolidation: on the role of brain-derived neurotrophic factor signaling in hippocampal-dependent learning. Learn Mem 2002; 9: 224-37.

9 Bliss TV, Gardner-Medwin AR. Long-lasting potentiation of synaptic transmission in the dentate area of the unanaestetized rabbit following stimulation of the perforant path. J Physiol 1973; 232 : 357-74.

10 Bliss TV, Lomo T. Long-lasting potentiation of synaptic transmission in the dentate area of the anaesthetized rabbit following stimulation of the perforant path. J Physiol 1973; 232: 331-56.

11 Bliss TV, Collingridge GL. A synaptic model of memory: long-term potentiation in the hippocampus. Nature 1993; 361: 31-9.

12 Chang L, Karin M. Mammalian MAP kinase signalling cascades. Nature 2001; 410 (6824): 37-40

13 Pearson G, Robinson F, Beers GT, Xu BE, Karandikar M, Berman K, et al. Mitogen-activated protein (MAP) kinase pathways: regulation and physiological functions. Endocr Rev 2001; 22: 153-83.

14 Selcher JC, Atkins CM, Trzaskos JM, Paylor R, Sweatt JD. A necessity for MAP kinase activation in mammalian spatial learning. Learn Mem 1999; 6: 478-90.

15 Blum S, Moore AN, Adams F, Dash PK. A mitogen-activated protein kinase cascade in the CA1/CA2 subfield of the dorsal hippocampus is essential for long-term spatial memory. J Neurosci 1999; 19: 3535-
44.

16 Miller CA, Marshall JF. Molecular substrates for retrieval and reconsolidation of cocaine-associated contextual memory. Neuron 2005; 47: 873-84.

17 Tapia-Arancibia L, Aliaga E, Silhol M, Arancibia S. New insights into brain BDNF function in normal aging and Alzheimer disease. Brain Res Rev 2008; 59: 201-20.

18 Li C, Yang J, Yu S, Chen N, Xue W, Hu J, et al. Triterpenoid saponins with neuroprotective effects from the roots of Polygala tenuifolia. Planta Med 2008; 74: 133-41.

19 Liu SL, Zhang JT. Effects of naloxone on L-clausenamide-induced long-term potentiation in dentate gyrus of anesthetized rats. Zhongguo Yao Li Xue Bao 1999; 20: 112-6.

20 Wang XY, Zhang JT. NO mediates ginsenoside Rg1-induced longterm potentiation in anesthetized rats. Acta Pharmacol Sin 2001; 22 : 1099-102.

21 Bouger PC, van der Staay FJ. Rats with scopolamine- or MK801-induced spatial discrimination deficits in the cone field task: animal models for impaired spatial orientation performance. Eur Neuropsychopharmacol 2005; 15: 331-46.

22 Wang R, Yan H, Tang XC. Progress in studies of huperzine A, a natural cholinesterase inhibitor from Chinese herbal medicine. Acta Pharmacol Sin 2006; 27: 1-26.

23 Richter-Levin G, Canevari L, Bliss TV. Long-term potentiation and glutamate release in the dentate gyrus: links to spatial learning. Behav Brain Res 1995; 66: 37-40.

24 Martin SJ, Grimwood PD, Morris RG. Synaptic plasticity and memory: an evaluation of the hypothesis. Annu Rev Neurosci 2000; 23: 649711.

25 Shalin SC, Hernandez CM, Dougherty MK, Morrison DK, Sweatt JD. Kinase suppressor of Ras1 compartmentalizes hippocampal signal transduction and subserves synaptic plasticity and memory formation. Neuron 2006; 50: 765-79.

26 Kelleher RJ III, Govindarajan A, Jung HY, Kang H, Tonegawa S. Translational control by MAPK signaling in long-term synaptic plasticity and memory. Cell 2004; 116: 467-79.

27 Sweatt JD. The neuronal MAP kinase cascade: a biochemical signal integration system subserving synaptic plasticity and memory. J Neurochem 2001; 76: 1-10.

28 Carew TJ. Molecular enhancement of memory formation. Neuron 2008; 16: 5-8.

29 Pang PT, Lu B. Regulation of late-phase LTP and long-term memory in normal and aging hippocampus: role of secreted proteins TPA and BDNF. Ageing Res Rev 2004; 3: 407-30.

30 Tully T, Bourtchouladze R, Scott R, Tallman J. Targeting the CREB pathway for memory enhancers. Nat Rev Drug Discov 2003; 2: $267-$ 77.

31 Fumagalli F, Racagni G, Riva MA. The expanding role of BDNF: a therapeutic target for Alzheimer's disease? Pharmacogenomics J 2006; 6: 8-15.

32 da Penha BM, Cooper J, Castren E, Zafra F, Sofroniew M, Thoenen $\mathrm{H}$, et al. Cholinergic regulation of brain-derived neurotrophic factor (BDNF) and nerve growth factor (NGF) but not neurotrophin-3 (NT-3) mRNA levels in the developing rat hippocampus. J Neurosci 1993; 13: 3818-26.

33 Lindefors N, Ernfors P, Falkenberg T, Persson H. Septal cholinergic afferents regulate expression of brain-derived neurotrophic factor and beta-nerve growth factor mRNA in rat hippocampus. Exp Brain Res 1992; 88: 78-90.

34 Tao X, Finkbeiner S, Arnold DB, Shaywitz AJ, Greenberg ME. $\mathrm{Ca}^{2+}$ influx regulates BDNF transcription by a CREB family transcription factor-dependent mechanism. Neuron 1998; 20: 709-26.

35 Jovanovic JN, Benfenati F, Siow YL, Sihra TS, Sanghera JS, Pelech SL, et al. Neurotrophins stimulate phosphorylation of synapsin I by MAP kinase and regulate synapsin I-actin interactions. Proc Natl Acad Sci USA 1996; 93: 3679-83. 